\title{
Atenuación de la respuesta cardíaca de defensa (RCD) como posible biomarcador de déficits motivacionales en depresión subclínica
}

\author{
Nieves Fuentes-Sánchez \\ nfuentes@uji.es \\ Miguel Escrig \\ ayuso@uji.es \\ Irene Jaén \\ ijaen@uji.es \\ Eva Cifre \\ cifre@uji.es \\ M. ${ }^{\text {a }}$ Carmen Pastor \\ mpastor@uji.es
}

\section{Resumen}

Las personas jóvenes pueden encontrarse en situaciones estresantes que provocan emociones negativas, tales como ansiedad o depresión, tanto en contextos de desempleo como de empleo. Precisamente, la respuesta cardíaca de defensa (RCD) ha sido ampliamente utilizada en el marco de la investigación del estrés, la ansiedad y la depresión. Esta respuesta - provocada por un estímulo aversivo, intenso e inesperado- combina componentes acelerativos y decelerativos, cuya significación psicológica se atribuye tanto a procesos atencionales como emocionales. En concreto, el segundo componente acelerativo se ha interpretado como un indicador de activación del sistema motivacional defensivo. La presente investigación tiene como objetivo analizar el patrón de RCD en una muestra de 71 jóvenes (39 mujeres) menores de 30 años en situaciones de empleo y desempleo. La tarea experimental consistió en la presentación de un ruido blanco $(500 \mathrm{~ms}, 105 \mathrm{~dB})$ tras un periodo de reposo de 6 minutos. Los componentes acelerativos y decelerativos se obtuvieron a partir de los $80 \mathrm{~s}$ posteriores al sonido aversivo. Al finalizar la sesión experimental, se administró una batería de cuestionarios, entre ellos el BDI (Beck Depression Inventory). Los resultados mostraron mayores puntuaciones en depresión y menor reactividad cardíaca en el segundo componente acelerativo en el grupo de jóvenes desempleados/as, comparado con el grupo de empleados/as. Nuestros datos sugieren que la segunda aceleración cardíaca podría considerarse como un biomarcador de posibles déficits en el sistema 
motivacional defensivo en poblaciones subclínicas con riesgo de sufrir depresión.

Palabras clave: depresión, sistema motivacional defensivo, respuesta cardíaca de defensa, jóvenes, desempleo.

\section{Abstract}

Young people can find themselves in stressful situations that cause negative emotions, such as anxiety or depression, in both unemployment and employment contexts. To this extent, the cardiac defense response (RCD) has been widely used in the framework of the investigation of stress, anxiety and depression. This response - produced by an aversive, intense and unexpected stimulus - is composed of accelerating and decelerating components, whose psychological significance is attributed to both attentional and emotional processes. In particular, the second accelerating component has been interpreted as an indicator of activation of the defensive motivational system. The present research aims to analyze the RCD pattern in a sample of 71 youths (39 female) under 30 years of age in situations of employment and unemployment. The experimental task consisted of the presentation of a white noise $(500 \mathrm{~ms}, 105 \mathrm{~dB})$ after a rest period of 6 minutes. Accelerating and decelerating components were obtained from the $80 \mathrm{~s}$ after the aversive sound. At the end of the experimental session, a battery of questionnaires was administered, including the BDI (Beck Depression Inventory). The results showed higher scores in depression and lower cardiac reactivity in the second accelerating component in the group of unemployed youths, compared to the group of employees. Our data suggest that the second cardiac acceleration might be considered as a biomarker of possible deficits in the defensive motivational system in subclinical populations at risk of depression.

Keywords: depression, defense motivation system, cardiac defense response, youths, unemployment.

\section{Introducción}

La depresión es uno de los trastornos psiquiátricos más frecuentes e incapacitantes. Numerosos estudios han relacionado la depresión con un incremento del riesgo de padecer enfermedades cardiovasculares (Brindle, Ginty y Conklin 2013; Carroll, Phillips, Hunt y Der 2007; Silvia, Nusbaum, Eddington, Beaty y Kwapil 2014). No obstante, los mecanismos subyacentes que relacionan ambas variables no están firmemente establecidos. A este respecto, la hipótesis tradicional de reactividad cardiovascular ante estímulos estresores propone que una reactividad cardíaca exagerada o prolongada predice enfermedades cardiovasculares (Souza et al. 2015). Por el contrario, teorías más recientes sugieren que la reactividad cardíaca atenuada también puede ser un marcador de un estado de salud deteriorado (Salomon, Clift, Karlsdottir y Rottenberg 2009; Salomon, Bylsma, White, Panaite y Rottenberg 2013; Souza et al. 2015). En este sentido, presentar unos niveles normales de reactividad cardíaca ante un estímulo estresor resulta completamente funcional y adaptativo, puesto que supone una movilización de energía necesaria para hacer frente a dicha estimulación. Por el contrario, mostrar una reactividad exagerada o atenuada podría ser desadaptativo en algunos contextos (Salomon, Bylsma, White, Panaite y Rottenberg 2013). La mayoría de literatura al respecto investiga la reactividad cardiovascular a través de la medición de la tasa cardíaca (Souza et al. 2015; Carroll, 
Phillips, Hunt y Der 2007) y la presión sanguínea (Carroll, Phillips, Hunt y Der 2007). Dichas medidas del sistema nervioso autónomo (SNA) reflejan una combinación de la actividad del sistema nervioso simpático (asociado con activación) y del sistema nervioso parasimpático (asociado con relajación) (Mauss y Robinson 2009).

Sin embargo, existe otra medida de reactividad cardíaca que nos puede proporcionar mayor información sobre la inervación simpática y parasimpática, además de proporcionarnos información sobre aspectos motivacionales (movilización de esfuerzos): la respuesta cardíaca de defensa (RCD). Dicha medida consiste en un patrón de reactividad cardíaca compuesto por dos componentes acelerativos (una aceleración de corta latencia y una segunda aceleración de larga latencia) y dos componentes decelerativos que aparecen secuencialmente durante los 80 segundos después de la presentación del estímulo intenso y aversivo (mayoritariamente auditivo). Este patrón está influenciado por mecanismos fisiológicos subyacentes tanto simpáticos como parasimpáticos. En concreto, la primera aceleración/deceleración es controlada por influencias parasimpáticas (es decir, inhibición durante la primera aceleración y activación durante la subsecuente deceleración). Por su parte, la segunda aceleración/deceleración es controlada tanto por la rama simpática como por la rama parasimpática actuando de manera recíproca, observándose activación simpática -acompañada por inhibición parasimpática- durante la segunda aceleración e inhibición simpática —acompañada por activación parasimpáticadu rante la segunda deceleración (Vila et al. 2007).

Tradicionalmente, este patrón autonómico se ha explicado desde dos aproximaciones teóricas. Por una parte, la aproximación cognitiva asume que los cambios cardíacos reflejan mecanismos atencionales y perceptivos. Desde esta aproximación se distingue entre el reflejo de orientación (una deceleración cardíaca), el cual facilita la atención al estímulo, y el reflejo de defensa (una aceleración cardíaca), el cual reduce la atención al estímulo aversivo como una forma de protección contra el estímulo amenazante (Vila et al. 2007). Por su parte, la aproximación motivacional asume que los cambios cardíacos en respuesta a los estímulos ambientales reflejan mecanismos metabólicos con el objetivo de proporcionar al organismo la energía necesaria para actuar. Cuando se requiere una respuesta activa, tanto psicológica como fisiológica, la reacción se acompañará de aceleración cardíaca, pero si es más apropiado responder con una conducta pasiva el organismo presentará una clara deceleración cardíaca (Vila et al. 2007).

Sin embargo, en los últimos años ambas tradiciones se han integrado dentro de un modelo teórico atencional-motivacional: el modelo de la cascada defensiva (Vila et al. 2009), el cual propone que las reacciones defensivas siguen un proceso secuencial con fases iniciales atencionales dirigidas a la detección y el análisis de la posible amenaza, seguidas de fases posteriores en las que predominan los factores motivacionales dirigidos a facilitar las acciones defensivas de lucha o huida (Blanchard y Blanchard 1989; Fanselow 1994; Lang, Davis y Öhman 2000) (véase la figura 1).

Desde esta perspectiva se plantea que la respuesta cardíaca de defensa está modulada tanto por factores atencionales como emocionales. Así, los distintos componentes de la respuesta parecen reflejar la sucesión de dos fases: 1) una primera fase atencional, dirigida a interrumpir la actividad y analizar el peligro potencial del estímulo, y 2) una fase motivacional, dirigida a preparar al organismo para la defensa activa. De esta forma, el patrón de respuesta cardíaca de defensa representa una transición de la atención (orientación al estímulo) a la acción (respuesta hacia el estímulo). La primera aceleración/deceleración implica la fase atencional, mientras que la segunda aceleración/deceleración refleja la fase motivacional, preparando al 
organismo para la defensa y permitiendo su recuperación en el caso de que el peligro no sea real (Vila et al. 2007).

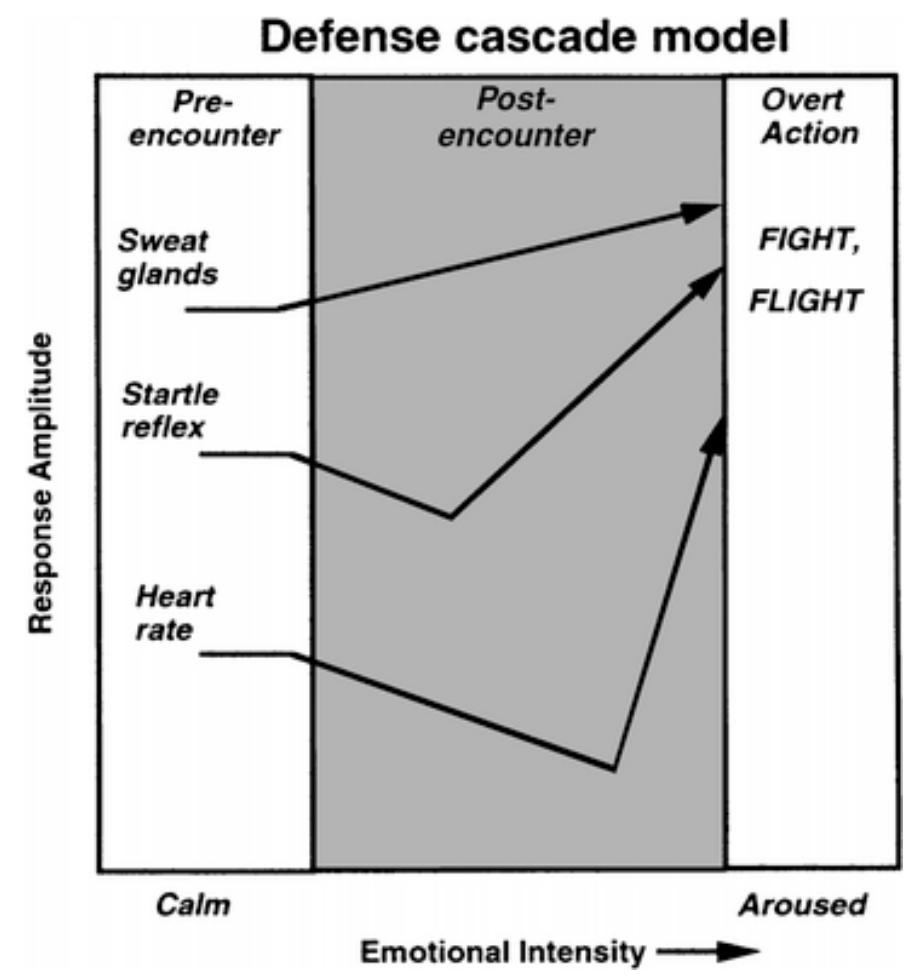

Figura 1. Modelo de la cascada defensiva (tomado de Bradley y Lang 2000).

Teniendo en cuenta este modelo, el segundo componente acelerativo —cuyo pico de máxima respuesta ocurre entre los 20 y 45 segundos posteriores a la presentación del estímulo intenso y aversivo- refleja la movilización de los recursos del organismo para dar una respuesta de afrontamiento, interpretándose como un buen indicador de la activación del sistema motivacional defensivo (López et al. 2016). Esta segunda aceleración está inervada mayoritariamente por el sistema nervioso simpático, lo que nos permite tener información directa sobre la movilización de esfuerzos (a mayor activación del sistema nervioso simpático, mayor movilización de esfuerzo). Por tanto, la RCD y, en concreto, la segunda aceleración, podría ser un buen biomarcador para evaluar de forma objetiva aspectos motivacionales subyacentes en diferentes trastornos clínicos como la depresión.

El presente trabajo de investigación pretende explorar la relación entre la respuesta cardíaca de defensa $(R C D)$ y la presencia de sintomatología depresiva en población sana. Para llevarlo a cabo, se comparan dos muestras de jóvenes en diferente situación laboral (empleados/as frente a desempleados/as). La selección de estas dos muestras se basó en nuestro interés por investigar estresores reales de la vida cotidiana, con el objetivo de dar una mayor validez ecológica a nuestro estudio. A este respecto, la literatura previa ha mostrado que existe una relación entre el desempleo y el incremento en el malestar psicológico asociado a la grave crisis económica que atravesó nuestro país (Gili, Roca, Basu, McKee y Stuckler 2012). Especialmente en el caso del desempleo prolongado, los y las jóvenes se encuentran con una situación amenazante y aversiva sostenida que puede provocar diferentes emociones negativas, tales como miedo, ansiedad o depresión. En esta línea, seleccionar ambas muestras nos permite obtener información sobre los posibles niveles de depresión y reactividad cardíaca asociados a una situación estresante de la vida diaria. De acuerdo con 
estudios previos, esperamos encontrar mayores niveles de depresión y una atenuación de la RCD en el grupo de desempleados/as, en comparación con el grupo de empleados/as.

\section{Método}

\section{Participantes}

La muestra del estudio estuvo conformada por 71 participantes, de los cuales 31 estaban en situación de desempleo $(51,61 \%$ mujeres $)$ y 40 en situación de empleo $(57,7 \%$ mujeres). Previo al estudio, se fijó como criterio que todos los y las participantes tuvieran un mínimo de dos años de experiencia laboral (aunando diferentes periodos) y un mínimo de 6 meses en situación de desempleo o empleo (para cada grupo, respectivamente). Para los análisis estadísticos se excluyeron 4 sujetos que se encontraban bajo tratamiento farmacológico, psiquiátrico o psicológico, por lo que finalmente la muestra se compuso de 67 personas $(55,22 \%$ mujeres), de las cuales 27 eran desempleadas y 40 empleadas en el momento del estudio. Los y las participantes tenían un mínimo de 18 años y un máximo de 30 años (Media $=27,80$, DT $=2,28)$, sin observarse diferencias de edad significativa entre ambos grupos $(p>0,233)$.

Antes de empezar el estudio, los y las participantes fimaron el consentimiento informado y recibieron una compensación económica por su participación tras finalizar la tarea. Además, el presente estudio forma parte de un proyecto de investigación más amplio aprobado por el Comité Deontológico de la Universitat Jaume I.

\section{Materiales}

Inventario de la Depresión de Beck [BDI-II] (Beck, Steer y Brown 1996): cuestionario compuesto por 21 ítems relacionados con síntomas depresivos (sentimientos de culpa, sentimientos físicos relacionados con la depresión, etc.) para evaluar la severidad de la depresión en una escala Likert de 4 puntos. Estos ítems corresponden con los criterios para el diagnóstico de los trastornos depresivos recogidos en el DSM-5. Un ejemplo de ítem es el siguiente: «Me siento triste gran parte del tiempo».

\section{Adquisición y reducción de datos fisiológicos}

Para la adquisición y el análisis de las distintas señales fisiológicas se utilizó un sistema de registro Biopac MP36 y el software Acqknowledge 4.1. La tasa cardíaca fue registrada mediante sensores de tamaño estándar $(8 \mathrm{~mm}$ de diámetro) colocados según la derivación II: dos electrodos activos —en la muñeca derecha y en el tobillo izquierdo- y un electrodo de tierra en el tobillo derecho. Previamente se limpiaron las zonas descritas frotando con un algodón impregnado en alcohol. Los valores de tasa cardíaca se expresaron como puntuaciones de cambio respecto a la media de los 15 segundos previos a la presentación del estímulo auditivo (línea de base). Con ello, se obtuvieron 80 valores (correspondientes a los 80 segundos tras la aparición del estímulo auditivo). Para facilitar el análisis estadístico, estos 80 valores de tasa cardíaca fueron reducidos a 10 valores, correspondientes a las medianas de los 10 intervalos siguientes: 2 intervalos de 3 segundos, 2 intervalos de 5 segundos, 3 intervalos de 7 segundos y 3 intervalos de 13 segundos. 


\section{Procedimiento}

El estudio se llevó a cabo en el laboratorio del grupo MPAGER situado en la Facultad de Ciencias Humanas y Sociales de la Universitat Jaume I. Previamente al experimento se contactó con los y las participantes para concretar fecha y hora a la que debían acudir y proporcionar algunas instrucciones que debían seguir antes de venir. Una vez en el laboratorio, se les hizo entrega del consentimiento informado en el que se les explicaba en qué consistía la sesión experimental. Tras firmar dicho documento, se procedía a la limpieza y colocación de los sensores para el registro del ECG y se leían las instrucciones en las que se les indicaba que debían estar en reposo, sin moverse, durante el tiempo que durara el experimento. No se les avisaba de la presentación del estímulo aversivo. Tras resolver cualquier duda que pudieran tener, se procedía a realizar el test de reactividad fisiológica para provocar la respuesta cardíaca de defensa (RCD). Esta tarea consistió en la presentación de un sonido intenso y aversivo (ruido blanco de $500 \mathrm{~ms}$ de duración, $105 \mathrm{~dB}$ y rise time instantáneo), administrado biauralmente a través de auriculares (Sennheiser HD 205), tras un periodo de reposo de 6 minutos (durante el cual se registraba la variable fisiológica). Después del sonido intenso se registraron los valores de ECG correspondientes a los 80 segundos posteriores. Tras finalizar la tarea, se administró una bateria de cuestionarios entre los que se encontraba el BDI-II. El resto de cuestionarios administrados no se incluyen en el presente estudio.

\section{Análisis estadístico}

Se realizaron análisis estadísticos para cada medida por separado (niveles de depresión y tasa cardíaca). En primer lugar, se realizó una prueba t para muestras independientes con el objetivo de examinar las diferencias entre empleados/as y desempleados/as en los niveles de depresión medidos por el BDI-II. Posteriormente, la respuesta cardíaca de defensa (CDR) fue examinada para toda la muestra a través del modelo lineal general (MLG) de medidas repetidas, 2 (grupo: empleados/as, desempleados/as) X 10 (medianas). El factor grupo fue incluido como factor entresujetos y las medianas como factor intra-sujeto. Se fijó el nivel de significación de $p<0,05$ usando la corrección Greenhouse-Geisser en los casos necesarios. Por último, se realizó un ANOVA de un factor con el objetivo de analizar las diferencias entre ambos grupos para cada una de las medianas. Los análisis estadísticos se llevaron a cabo mediante el software SPSS 24.

\section{Resultados}

Niveles de depresión en empleados/as y desempleados/as

La prueba t mostró diferencias significativas entre empleados/as y desempleados/as en los niveles de depresión evaluados con el BDI-II, $t(65)=2,91, p<0,005, d=0,71$. En concreto, los/las desempleados/as reportaron mayores nivelesde depresión $(M=13,15, S D=7,77)$ en comparación con los/las empleados/as $(M=8, S D=6,64)$.

\section{Patrón de respuesta cardíaca de defensa (RCD)}

El modelo lineal general (MLG) para toda la muestra mostró un efecto significativo del factor Mediana, $F(9,68)=21,67, p<0,0001, \eta^{2} p=0,24$, confirmando la presencia de un patrón típico de RCD con un primer componente acelerativo y un posterior componente decelerativo (véase la figura 2). La interacción entre el grupo 
(empleados/as frente a desempleados/as) $x$ mediana no fue significativo, $F(9$, 68) $=1,02, p=0,43, \eta^{2} p=0,01$, lo que indica que ambos grupos presentaban un patrón de RCD similar con los dos componentes acelerativos y decelerativos de forma secuencial (véase la figura 2).

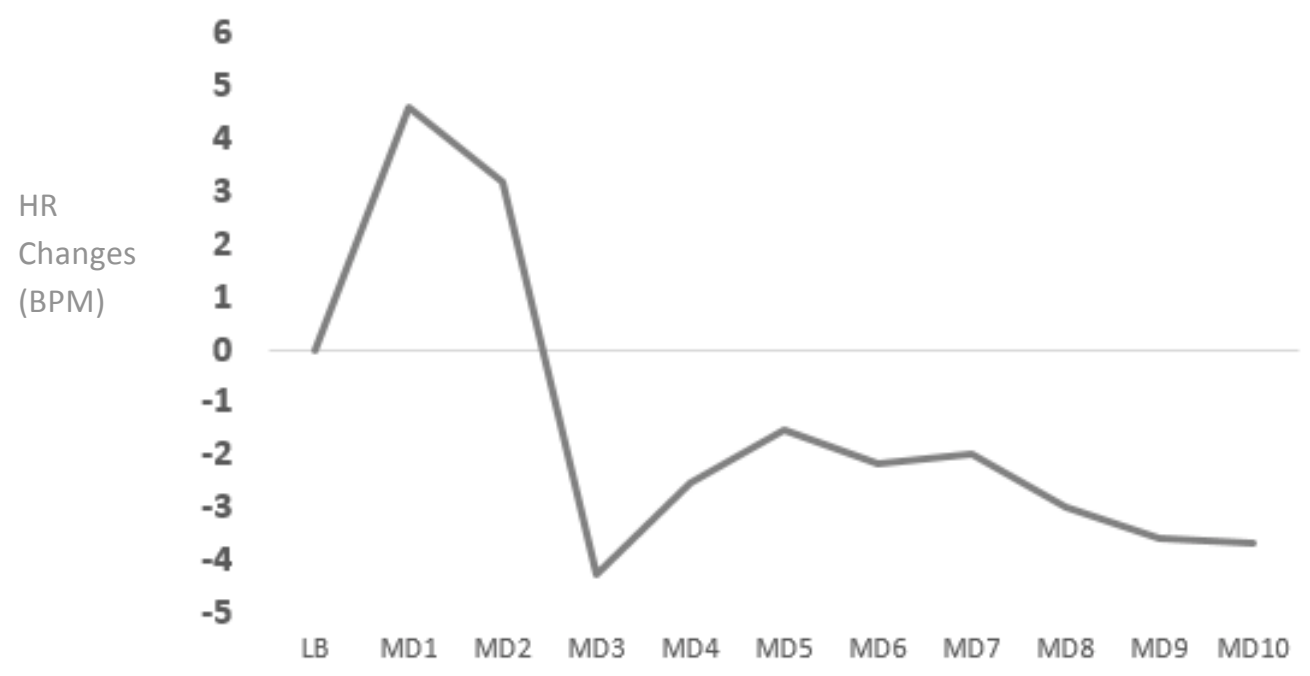

Figura 2. Patrón de respuesta cardíaca de defensa (RCD) para toda la muestra

Posteriormente, se realizó un ANOVA de un factor para analizar las diferencias entre ambos grupos para cada una de las medianas. El ANOVA mostró diferencias significativas entre empleados/as y desempleados/as únicamente en la mediana $7, F$ $(1,68)=3,8, p=0,05, d=0,48$ (véanse la figura 3 y la tabla 1 ).

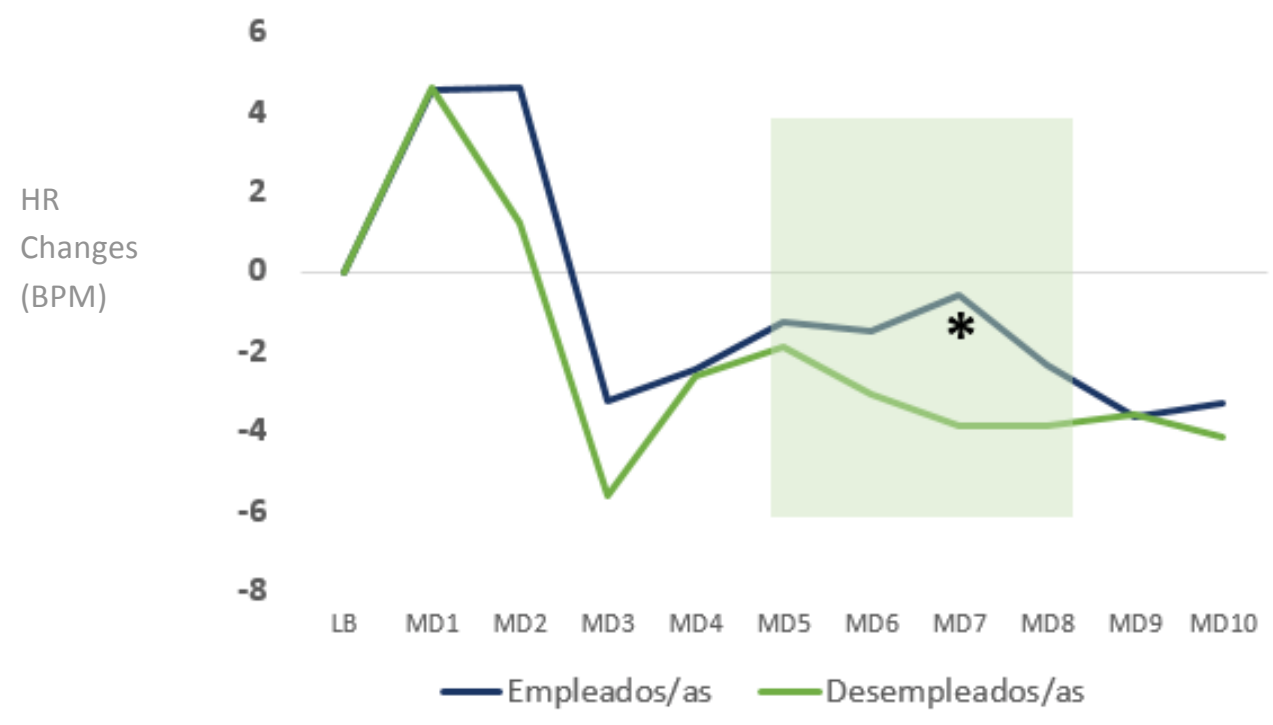

Figura 3. Patrón de RCD para la muestra de empleados/as y desempleados/as. 
Tabla 1.

Medias (M), desviaciones típicas (SD) y ANOVA para cada mediana.

\begin{tabular}{llll}
\hline & $\begin{array}{l}\text { Empleados/as } \\
(\mathrm{M}, \mathrm{SD})\end{array}$ & $\begin{array}{l}\text { Desempleados/as } \\
(\mathrm{M}, \mathrm{SD})\end{array}$ & Anova de un factor \\
\hline Md1 & $4,58(5,05)$ & $4,59(10,01)$ & $F<1$ \\
Md2 & $4,63(9,54)$ & $1,25(8,59)$ & $F(1,68)=2,35, p=0,13$ \\
Md3 & $-3,24(7,17)$ & $-5,64(8,83)$ & $F(1,68)=1,57, p=0,22$ \\
Md4 & $-2,45(6,20)$ & $-2,64(7,73)$ & $F<1$ \\
Md5 & $-1,24(6,65)$ & $-1,91(5,65)$ & $F<1$ \\
Md6 & $-1,526,64$ & $-3,05(5,99)$ & $F<1$ \\
Md7 &,$- 58(8,17)$ & $-3,88(5,00)$ & $F(1,68)=3,81, p=0,05^{*}$ \\
Md8 & $-2,33(5,73)$ & $-3,85(4,20)$ & $F(1,68)=1,51, p=0,22$ \\
Md9 & $-3,62(4,80)$ & $-3,57(3,57)$ & $F<1$ \\
Md10 & $-3,30(4,05)$ & $-4,16(2,74)$ & $F(1,68)=1,02, p=0,32$ \\
\hline
\end{tabular}

\section{Discusión y conclusiones}

El presente estudio pretendía explorar la relación entre los niveles de depresión y la reactividad cardíaca. Nuestros hallazgos muestran diferencias entre el grupo de empleados/as y desempleados/as en los niveles de depresión. En concreto, el grupo de desempleados/as mostró un mayor nivel de depresión. Este resultado va en la línea de la hipótesis propuesta y de los resultados previos (Blanch 2011), los cuales sugieren que la situación de desempleo produce repercusiones psicosociales entre las que destaca el malestar, la incertidumbre, el pesimismo, la depresión, el sentimiento de inseguridad, la desesperanza y la frustración, entre otros (Del Pozo, Ruíz, Pardo y San Martín 2002).

Por otra parte, los datos del presente estudio han permitido replicar el patrón de RCD tanto para la muestra conjunta de empleados/as y desempleados/as como para cada grupo experimental por separado, en el que se observan claramente dos componentes acelerativos y decelerativos de manera secuencial, de acuerdo con los resultados de trabajos previos (Vila et al. 2007; Vila et al. 2003). Además, cuando se analizan las diferencias entre ambos grupos, se observa una atenuación de la RCD en los/las desempleados/as en la segunda aceleración, en la línea de los resultados de investigaciones previas (Salomon, Clift, Karlsdottir y Rottenberg 2009; Salomon, Bylsma, White, Panaite y Rottenberg 2013). A partir de este dato, nos planteamos una serie de cuestiones relevantes para futuras investigaciones. En primer lugar, si la atenuación de la reactividad cardíaca podría ser un biomarcador del riesgo de depresión en población sana que todavía no ha desarrollado sintomatología clínica (Salomon, Bylsma, White, Panaite y Rottenberg 2013). Los resultados de estudios previos van en la línea de dicha hipótesis (véase, por ejemplo, Phillips, Hunt, Der y Carroll 2011; Rottenberg, Salomon, Gross y Gotlib 2005; Salomon, Bylsma, White, Panaite y Rottenberg 2013). Concretamente, muestran que una reactividad atenuada parece asociarse con futura sintomatología depresiva en individuos no diagnosticados actualmente con trastorno depresivo mayor, sugiriendo que la reactividad atenuada puede indicar riesgo de padecer depresión clínica y no necesariamente depresión actual (Salomon, Bylsma, White, Panaite y Rottenberg 2013). Otra de las cuestiones es si existen procesos psicológicos que subyacen a la relación entre esta atenuación cardíaca y la depresión. En este sentido, Lovallo (2011) y Carroll, Phillips, Hunt y Der (2007) argumentaron que la reactividad cardíaca atenuada podría deberse a una desregulación del sistema emocional y motivacional. En el contexto de laboratorio podemos evaluar esta desregulación motivacional a través de la movilización de 
esfuerzo. Una de las formas de evaluar esa movilización de esfuerzo es a través de la activación del sistema nervioso simpático (concretamente, a partir de la información proporcionada por el segundo componente acelerativo de la RCD). A este respecto, nuestros datos muestran una menor activación del sistema nervioso simpático (segun da aceleración) en el grupo con mayores niveles de depresión, lo que podría estar sugiriendo una menor movilización de esfuerzo y, por tanto, estos datos proporcionarían un apoyo tentativo a la idea de utilizar la RCD como un posible biomarcador de déficits motivacionales en depresión. En esta línea, se han encontrado resultados similares en otros trastornos relacionados con una desregulación del sistema motivacional y emocional, como es el caso de los trastornos alimenticios (Ginty, Phillips, Higgs, Heaney y Carroll 2012) o del riesgo de adicción (Pankin, Dickensheets, Nixon y Lovallo 2002).

En resumen, nuestros datos muestran que individuos con un mayor nivel de depresión presentan una atenuación de la RCD en el segundo componente acelerativo y, por tanto, una menor movilización de recursos, lo que podría estar indicando un posible déficit motivacional (medido a través de la activación del sistema nervioso simpático). Además, al ser una muestra subclínica (es decir, ningún participante está diagnosticado con trastorno depresivo mayor), la atenuación cardíaca podría estar actuando como un biomarcador del riesgo de depresión. Aunque estos resultados van en la línea de hallazgos previos, cabe resaltar la necesidad de nuevos estudios que confirmen y repliquen nuestros datos. En este sentido, se necesitan estudios longitudinales con muestras subclínicas para comprobar si realmente la atenuación cardíaca predice la depresión en el futuro. Por otra parte, son necesarios más trabajos experimentales que investiguen los procesos psicológicos subyacentes a dicha atenuación cardíaca.

\section{Referencias bibliográficas}

Beck. Aaron T., Robert A. Steer y Gregory K. Brown. 1996. Manual for the Beck Depression Inventory-II. San Antonio, TX: Psychological Corporation.

Blanch, Josep M. 2011. «La psicología del trabajo ante la crisis del empleo». Infocop 55: 7-11.

Blanchard, Robert J. y D. Caroline Blanchard. 1989. «Attack and defense in rodents as ethoexperimental models for the study of emotion». Progress in NeuroPsychopharmacology and Biological Psychiatry 13: 3-4.

Bradley, Margaret M. y Peter J. Lang. 2000. «Affective reactions to acoustic stimuli». Psychophysiology 37: 204-215.

Brindle, Ryan C., Annie T. Ginty y Sarah M. Conklin. 2013. «Is the association between depression and blunted cardiovascular stress reactions mediated by perceptions of stress?». International Journal of Psychophysiology 90(1): 66-72.

Carroll, Douglas, Anna C. Phillips, Kate Hunt y Geoff Der. 2007. «Symptoms of depression and cardiovascular reactions to acute psychological stress: Evidence from a population study». Biological Psychology 75: 68-74.

Del Pozo, Juan A., Miguel A. Ruíz, Antonio Pardo y Rafael San Martín. 2002. «Efectos de la duración del desempleo entre los desempleados». Psicothema 14: 440443.

Fanselow, Michael S. 1994. "Neural organization of the defense behaviour system responsible for fear». Psychonomic Bulletin and Review 1: 429-438.

Gili, Margalida, Miquel Roca, Sanjay Basu, Martin McKee y David Stucjler. 2012. "The mental healh risk of economic crisis in Spain: evidence from primary care centres, 2006 and 2010». The European Journal of Public Health 23(1): 103108. 
Ginty, Annie T., Anna C. Phillips, Suzanne Higgs, Jennifer Heaney y Douglas Carroll. 2012. «Disordered eating behaviour is associated with blunted cortisol and cardiovascular reactions to acute psychological stress». Psychoneuroendocrinology 37(5): 715-724.

Lang, Peter J., Michael Davis y Arne Öhman. 2000. «Fear and anxiety: Animal models and human cognitive psychophysiology». Journal of Affective Disorders 61: 137-159.

López, Raúl, Rosario Poy, Pilar Segarra, Àngels Esteller, Alicia Fonfría, Pablo Ribes, Carlos Ventura y Javier Moltó. 2016. "Gender-specific effects of trait anxiety on the cardiac defense response». Personality and Individual Differences 96: 243247.

Lovallo, William R. 2011. "Do low levels of stress reactivity signal poor states of health?». Biological Psychology 86(2): 121-128.

Mauss, Iris B. y Michael C. Robinson. 2009. "Measures of emotion: A review». Cognition and Emotion 23(2): 209-237.

Panknin, Tera L., Stacey L. Dickensheets, Sara Jo Nixon y William R. Lovallo. 2002. «Attenuated heart rate responses to public speaking in individuals with alcohol dependence». Alcoholism, Clinical and Experimental Research 26(6): 841-847.

Phillips, Anna C., Kate Hunt, Geoff Der y Douglas Carroll. 2011. «Blunted cardiac reactions to acute psychological stress predict symptoms of depression five years later: evidence from a large community study». Psychophysiology 48(1): 142-148.

Rottenberg, Jonathan, Kristen Salomon, James J. Gross e lan H. Gotlib. 2005. «Vagal withdrawal to sad film predicts subsequent recovery from depression». Psychophysiology 42: 277-281.

Salomon, Kristen, April Clift, Mardis Karlsdottír y Jonathan Rottenberg. 2009. «Major depressive disorder is associated with attenuated cardiovascular reactivity and impaired recovery among those free of cardiovascular disease». Health Psychology 28: 157-165.

Salomon, Kristen, Lauren M. Bylsma, Kristi E. White, Vanessa Panaite y Jonathan Rottenberg. 2013. "Is blunted cardiovascular reactivity in depression moodstate dependent? A comparison of major depressive disorder remitted depression and healthy controls». International Journal of Psychophysiology 90(1): 50-57.

Silvia, Paul J., Emily C. Nusbaum, Kari M. Eddington, Roger E. Beaty y Thomas R. Kwapil. 2014. «Effort deficits and depression: The influence of anhedonic depressive symptoms on cardiac autonomic activity during a mental challenge». Motivation and Emotion 38(6): 779-789.

Souza, Gabriela Guerra Leal, Ana Carolina Ferraz Mendonça-de-Souza, Antonio F. A. Duarte, Nastassja L. Fischer, Wanderson F. Souza, Evandro Silva Freire Coutinho, Ivan Figueira y Eliane Volchan. 2015. «Blunted cardiac reactivity to psychological stress associated with higher trait anxiety: a study in peacekeepers». BMC Neuroscience 16: 81.

Vila, Jaime, María Carmen Fernández, Joaquín Pegalajar, María Nieves Vera, Humbelina Robles, María Nieves Pérez-Marfil, María B. Sánchez, Isabel Ramírez y Elisabeth Ruiz-Padial. 2003. "A new look at Cardiac Defense: Attention or Emotion?». The Spanish Journal of Psychology 6: 60-78.

Vila, Jaime, Pedro Guerra, Miguel Ángel Muñoz, Cynthia Vico, María Isabel Viedmadel Jesús, Luis Carlos Delgado, Pandelis Perakakis, Elisabeth Kley, Jose Luis Mata y Sonia Rodriguez. 2007. "Cardiac defense\%: From attention to action». International Journal of Psychophysiology 66: 169-182.

Vila, Jaime, Pedro Guerra, Miguel Ángel Muñoz, Pandelis Perakakis, Luis Carlos Delgado, Marlen Figueroa y Sofía Mohamed. 2009. "La dinámica del miedo: la cascada defensiva». Escritos de Psicología 3(1): 37-42. 